\title{
Promising properties of ALD boron nitride nanotube mat for water purification ${ }^{1}$
}

\author{
W. Hao, C. Marichy* and A. Brioude \\ Univ Lyon, Université Claude Bernard Lyon 1, Laboratoire des Multimatériaux et Interfaces, UMR CNRS \\ 5615, F-69622 Villeurbanne, France \\ *Corresponding author: catherine.marichy@univ-lyon1.fr
}

\begin{abstract}
From low cost template made of electrospun polyacrylonitrile fibers, highly stable boron nitride nanotube (BNNT) mat is fabricated using low temperature atomic layer deposition (ALD) of BN. Based on polymer derived ceramics chemistry, this ALD process permits preparing high quality BNNTs that show superhydrophocity, stability over a month in air and various $\mathrm{pH}$ conditions as well as excellent performances in water treatment. In particular, the obtained material presents high ability as separation filter for oil from aqueous phase in immiscible mixture and emulsion. The BNNT mat display also excellent renewable performances for various organic solvents and oils absorption making this unwoven material a highly promising material for efficient water purification and treatment.
\end{abstract}

Keywords: Atomic Layer Deposition; h-BN, Water treatment, coating, nanostructures, thin film, PDCs

\section{Introduction}

Water covers about $71 \%$ of the earth in which an important percentage is either salty or polluted water. Heavy metals, organics as oils and grease, and micro-/nano-particles represent an important part of the toxic pollutants. Lack of available drinkable water resources is one of the major concerns of the world population and water treatment thus generates intensive research. Recently, compared to the conventional methods and materials to purify water, nanomaterials appear extremely promising for the water treatment. ${ }^{1-3}$ Until now, various kinds of nanostructures have been reported, such as $\mathrm{TiO}_{2}$ composites, ${ }^{4}$ $\mathrm{Cu}_{2} \mathrm{O}$ nanoparticles, ${ }^{5}$ amyloid-carbon hybrid membranes, ${ }^{6}$ PDVB-modified mesh based materials, ${ }^{7}$ carbon nanotubes, ${ }^{8,9}$ boron nitride based materials, ${ }^{10-13}$ etc. However, use of such nanomaterials might cause a second contamination by nanoscale pollutants into the filtrated water. Hexagonal boron nitride (h-BN) is a 2D material with strong in-plane covalent bond and weak out-of-plane Van der Waals bond displaying high thermal and chemical stability, low density and hydrophobicity. ${ }^{14,15}$ These specific characteristics make h-BN a suited ceramics for water purification potentially preventing second contamination, compared to other materials such as $\mathrm{TiO}_{2}, \mathrm{ZnO}$ or $\mathrm{CuO}$ that might more easily dissolve, disperse or

\footnotetext{
${ }^{1}$ Some references are missing compared to the published version
} 
undergo chemical change into an aqueous media than BN. In particular a recent review, C. H. Lee $e t a l^{3}$ report that boron nitride nanotubes (BNNTs) are one of the nanomaterials that might be used for water treatment without any second contamination risk. On one hand, BN nanostructures such as foam-like porous monoliths, ${ }^{16}$ porous nanosheets, ${ }^{17}$ ultrathin fibrous nanonets, ${ }^{11}$ activated nano or microribbons, ${ }^{18,19}$ nanosheet-structured spheres,${ }^{20}$ particles and nanosheets, ${ }^{10}$ free-standing membranes, ${ }^{21}$ have been reported with efficient oil absorption and dye or heavy metal ions removal properties. On the other hand, freestanding $\mathrm{BN}$ nanosheet films ${ }^{12}$ and $\mathrm{BN}$ nanotube-coated stainless steel meshes ${ }^{22}$ have demonstrated efficient and ultrafast separation of immiscible oil/water mixture, while composites materials such as BN/graphite oxide, ${ }^{23}$ porous $\mathrm{BN}$ nanosheet/polymer membrane, ${ }^{24}$ and porous $\mathrm{BN}$ nanosheet/polyvinylidene fluoride ${ }^{25}$ have also been prepared and their water purification abilities investigated. Despite the variety of nano- hetero-structures already available, it remains indispensable to fabricate novel versatile materials easy to handle such as mats and large clothes, showing efficiency under multiple purification conditions, meaning adsorption of oil or organic solvents absorption and separation of immiscible oil/water mixtures as well as emulsions, etc.

It has been proved that with proper organization, combining roughness effect and intrinsic hydrophobicity of the material, boron nitride nano or micro structures present super hydrophobicity. ${ }^{156-28}$ Synthesis of such nanomaterials requires controllable and tunable approach. In particular, due to its simplicity, accuracy and versatility, atomic layer deposition (ALD) has demonstrated to be a very suited technique for fabricating nano-/hetero-structures. ${ }^{29-31}$ Based on polymeric template, ALD fabrication of high stable unwoven BNNT mats is reported here. Owing to their low cost, electrospun polymeric nano- or microfibers are used as sacrificial templates to produce superhydrophobic h-BN structures using a two-step ALD of BN based on polymer derived ceramics (PDCs) chemistry. ${ }^{32}$ Due to the low deposition temperature $\left(80^{\circ} \mathrm{C}\right)$ and no ammonia use, this process allows coating of polymeric substrates which are thus removed during the second step consisting in high temperature densification. Moreover, ALD could provide a conformal and uniform depositing manner to tune the wall thickness and diameter of the target nano or micro tubes. ${ }^{33}$ it should be pointed out that the capillarity of the nano or micro tubes could enhance the oil/water separation process. ${ }^{34}$

Herein, structures and properties of the obtained unwoven BNNT mats are investigated in details. Especially they exhibit outstanding hydrophobicity and lipophilicity properties as well as exceptional stability over a period of one month. Neither air exposure nor drastic $\mathrm{pH}$ conditions seem alter significantly their properties, which is a sign of very good BN quality. The potential of BNNTs in absorption-based oil/water separation, filtration-based immiscible oil/water mixture separation, and water- 
in-oil emulsion filtration is demonstrated making this material highly promising for water purification. Importance of the nanotube size toward its soaking properties of various organics/oil is also highlighted.

\section{Experimental section}

\section{Materials}

PAN (polyacrylonitrile) was obtained from Sigma-Aldrich, average $M_{w}=150,000$. DMF (N,NDimethylformamide) was obtained from Sigma-Aldrich (extra-dry, water $<50 \mathrm{ppm}$ ). Hexamethyldisilazane ( $\mathrm{NH}\left(\mathrm{SiMe}_{3}\right)_{2}$, HMDS) was purchase from Carl Roth (98\%). PAN, DMF and HMDS were used without further purification. Trichloroborazine $\left(\mathrm{B}_{3} \mathrm{~N}_{3} \mathrm{H}_{3} \mathrm{Cl}_{3}, \mathrm{TCB}\right)$ previously synthesized at the laboratory according to ref. ${ }^{35}$ was purified by sublimation before filling of the canister.

\section{Fabrication of unwoven BNNTs}

Unwoven PAN fibers are fabricated using electrospinning according to ref. ${ }^{36,37}$ The polymer mat obtained is then used as template for ALD. Briefly, the ALD process was performed in a simple home-made ALD glassware set-up coupled with typical vacuum/Ar Schlenk line. It consists of a glass tube connected on one side to two stopcocks that are each linked to one of the reactant bubblers, respectively, and on the other side toward vacuum via the Schlenk line. Before starting the deposition, the system was purged using three vacuum/Ar cycles and then vacuumed for $1 \mathrm{~h}$ at least. Depositions took place at $80{ }^{\circ} \mathrm{C}$. TCB was kept in a glass bubbler at $70{ }^{\circ} \mathrm{C}$ to be sublimated, while HMDS was maintained at room temperature $\left(25^{\circ} \mathrm{C}\right)$ inside a glass bubbler. Each precursor was sequentially introduced into the reactor with pulse of 2 and $3 \mathrm{~s}$, respectively, and separated to each other by a purge under Ar flow for $30 \mathrm{~s}$. The cycle number was fixed at 100 cycles. As-deposited thin films were post-annealed in a tubular furnace under $\mathrm{Ar}$ atmosphere up to $600{ }^{\circ} \mathrm{C}$ for $1 \mathrm{~h}$, and then under $\mathrm{NH}_{3}$ at $1400{ }^{\circ} \mathrm{C}$ for $3 \mathrm{~h}$ to remove the template. No exposure to air was realized before annealing. All transfers were performed under controlled atmosphere. The processing steps are represented in Figure 1. 


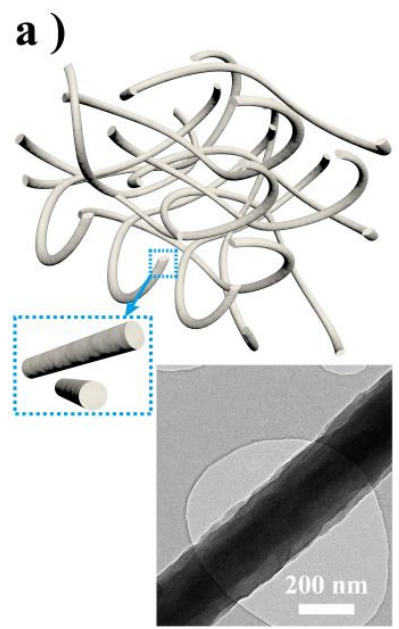

Starting electrospun PAN fiber mat

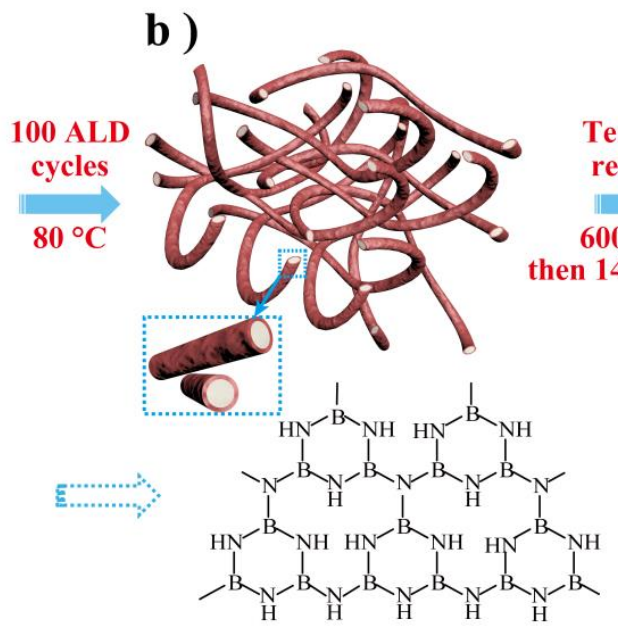

Polyborazylene coated PAN fibers c)

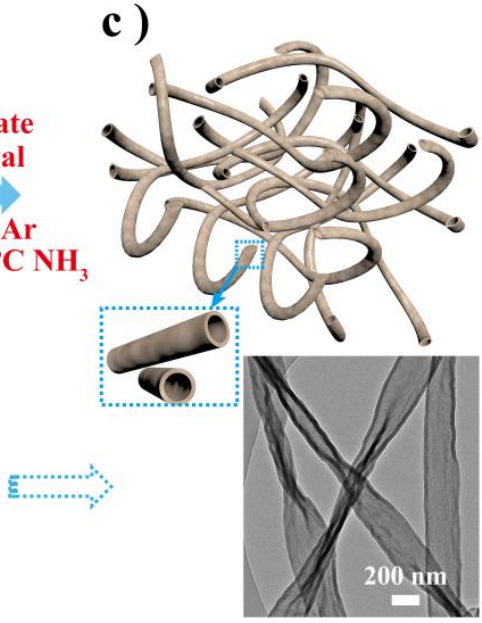

BN nanotube mat

Figure 1. Scheme of the processing steps: a) starting electrospun PAN fibers; b) polyborazylene coated PAN fibers after 100 ALD cycles at $80^{\circ} \mathrm{C}$; c) BN nanotube mat after the template removal using heat treatment.

\section{Sample characterizations}

The coated-structures were characterized by scanning electron microscopy (SEM) and energy dispersive X-ray spectroscopy (EDXS) using a Zeiss Merlin VP compact microscope operated at $5 \mathrm{kV}$ and equipped with a SDD OXFORD X-Max EDXS detector. Samples were prepared without extra carbon or platinum coating. The BNNTs were also characterized by transmission electron microscopy (TEM) and high resolution TEM (HRTEM) using a JEOL 2100F microscope operated at $200 \mathrm{kV}$ and equipped of silicon drift detector (Oxford Instrument) for EDXS. TEM investigations of BN nanotubes were carried out on copper TEM grids with a holey carbon support film. The tubes were deposited on TEM grids from alcohol dispersion after a sonication step for $5 \mathrm{~min}$. Fourier transform infrared spectroscopy (FTIR) in attenuated total reflection mode was realized on the obtained BNNTs using a PerkinElmer Lambda 365 Fourier transform infrared spectroscope. The wetting properties were characterized with depositing $7 \mu$ of deionized water with using a Krüss Easydrop. Contact angles using aqueous solutions with $\mathrm{pH}$ varying from 0 to 14 and organic solvent such as toluene and dichloromethane were also measured.

\section{Water treatment: oil-organic/water separation}

\section{Absorption approach}

Ability of BNNT mat to remove various oils and organic solvents from water by absorption process was first tested by adding the mat into a glass dish containing few drops of used engine oil floating on top of water. The absorption capacity of the fabricated h-BNNT mat was then evaluated toward five different 
kinds of organics and oils with different densities and viscosities meaning toluene and dichloromethane solvent as well as pump, mineral and used engine oils. Difference in weight between dry and just after immersion into the pure compound of unwoven BNNTs is used as absorption capacity measurement. To ensure saturation of the material, immersion for $48 \mathrm{~h}$ into the organics or oils adsorption was realized. Weightings were done rapidly to avoid/minimized evaporation of tested organic solvent, while $5 \mathrm{~s}$ "cleaning" using a tissue were used in the case of oil adsorption to ensure that no extra high viscosity oil drops could influence the measurements. The regeneration of the BN material is realized using heat treatment at $600{ }^{\circ} \mathrm{C}$ under $\mathrm{N}_{2}$ and until $1400{ }^{\circ} \mathrm{C}$ for $2 \mathrm{~h}$ under $\mathrm{NH}_{3}$ to burn solvents and oils while preventing BNNT oxidation. The reuse ability of the unwoven BNNTs was tested by using at least 5 times the same fibers.

\section{Filtration approach}

Unwoven BNNT fibers were employed as filter for solution filtration using a home-made system (in Electronic Supporting Information (ESI) Figure S1). Capability of the filter was investigated using two types of mixture: immiscible mixture and emulsion. Surfactant free biphasic solution made of $5 \mathrm{ml} \mathrm{pH} 9$ aqueous solution and $15 \mathrm{ml}$ of dichloromethane was prepared. Bromothymol blue was used as $\mathrm{pH}$ indicator of the aqueous phase. Water-in-chloroform emulsion was prepared by mixing $1 \mathrm{~mL}$ water and $100 \mathrm{~mL}$ chloroform with $0.253 \mathrm{~g}$ Span80 surfactant. The preparation was maintained under vigorous stirring for $48 \mathrm{~h}$. The emulsions are stable for at least 7 days.

The presence of eventual BNNT contaminant into the purified water phase was checked using SEM by dropping $15 \mu \mathrm{L}$ of water onto silicon substrates.

\section{Results and discussions}

\section{Characterization of obtained $\mathrm{BN}$ nanotubes}

Unwoven h-BN nanotubes are successfully fabricated from electrospun PAN fiber mat using a two-step ALD process of boron nitride, consisting in deposition of pre-ceramic polyborazylene on electrospun PAN fibers template at low temperature $\left(<100{ }^{\circ} \mathrm{C}\right)$ and subsequent thermal annealing process to convert preceramic coatings into dense BN nanotubes. Average diameter of BNNTs and their wall thickness are controlled by simple tuning of, respectively, the initial PAN fiber diameter and the number of ALD cycles performed. The whole polymer fibers are covered in uniform and conformal manner by a thin ALD film. After heat treatment to densify the layer and remove the template, unwoven mat (Inset Figure 1a), made of 3D crosslinked BNNTs that display similar macroscopic features than that the starting electrospun PAN fibers (ESI Figure S2), is obtained as shown in Figure 2a. Due to its nano-structuration, such cloth-like material presents intrinsic surface roughness and porosity which are suited for further filtration application. 
SEM imaging (Figure 2b) reveals homogenous tubes with an average diameter of $250 \mathrm{~nm}$. The corresponding EDXS spectrum (inset in Figure $2 \mathbf{b}$ ) confirms the presence of $\mathrm{B}$ and $\mathrm{N}$ as the main elements with an atomic $\mathrm{B} / \mathrm{N}$ ratio of 1.01 , regardless a small amount of $\mathrm{C}$ and $\mathrm{O}$ elements attributed to the supporting carbon tape and contamination in air. The fabricated BN nanotubes present a uniform wall thickness of $28 \mathrm{~nm}$ along their whole length as nicely visible in low magnification TEM image (Figure 2c). The growth per cycle (GPC) on PAN fibers is thus $0.28 \mathrm{~nm} /$ cycle. It should be pointed out that this value is higher than that previously reported on $\mathrm{SiO}_{2} / \mathrm{Si}$ wafer. ${ }^{32}$ This might be attributed to precursor diffusion into the polymer template, phenomenon previously reported into the literature during ALD coating onto polymeric materials. ${ }^{39,40}$ In particular, the group of Knez develop vapor phase infiltration (VPI) and multiple pulsed vapor phase infiltration (MPI) to improve the thermal and mechanical properties of polymers. $^{41,42}$

Microstructure and crystallinity of the fabricated material are investigated using high resolution TEM (Figure 2d,e,f). Similarly to analysis under SEM mode, EDXS spectrum recorded with TEM (Figure 2e) of BNNT demonstrates formation of BN. Observed $\mathrm{C}$ and $\mathrm{O}$ elements are attributed to holey carbon TEM grid and preparation process in air. Lattice fringes of $0.33 \mathrm{~nm}$ corresponding to c-axis of hexagonal $\mathrm{BN}$ are nicely observed in HRTEM image of a tube wall (Figure 2d). Despite evidence of layered structure, structural disorder is noted sign of mix of h-BN and turbostratic BN (t-BN) as previously reported with use of PDCs route. ${ }^{43}$ HRTEM image recorded from open-end of one tube (Figure 2f) exhibits randomly oriented planes as well as stacking ordering distance of $0.33 \mathrm{~nm}$. The corresponding selected area (yellow frame area in Figure 1f) Fast Fourier transform (FFT) image (inset in Figure 2f) displays distinct ring pattern highlighting the polycrystalline structure of the obtained BNNTs.

FTIR spectrum of BN nanotubes, shown in Figure S2a (ESI), exhibit the typical signature of BN with strong B-N in plane vibrational $\left(1363 \mathrm{~cm}^{-1}\right)$ and B-N-B out-of-plane bending $\left(804 \mathrm{~cm}^{-1}\right)$ bands without presence of B-OH $\left(3419 \mathrm{~cm}^{-1}\right)$ or B-NH $2\left(3218 \mathrm{~cm}^{-1}\right)$ bonds. ${ }^{18}$ 


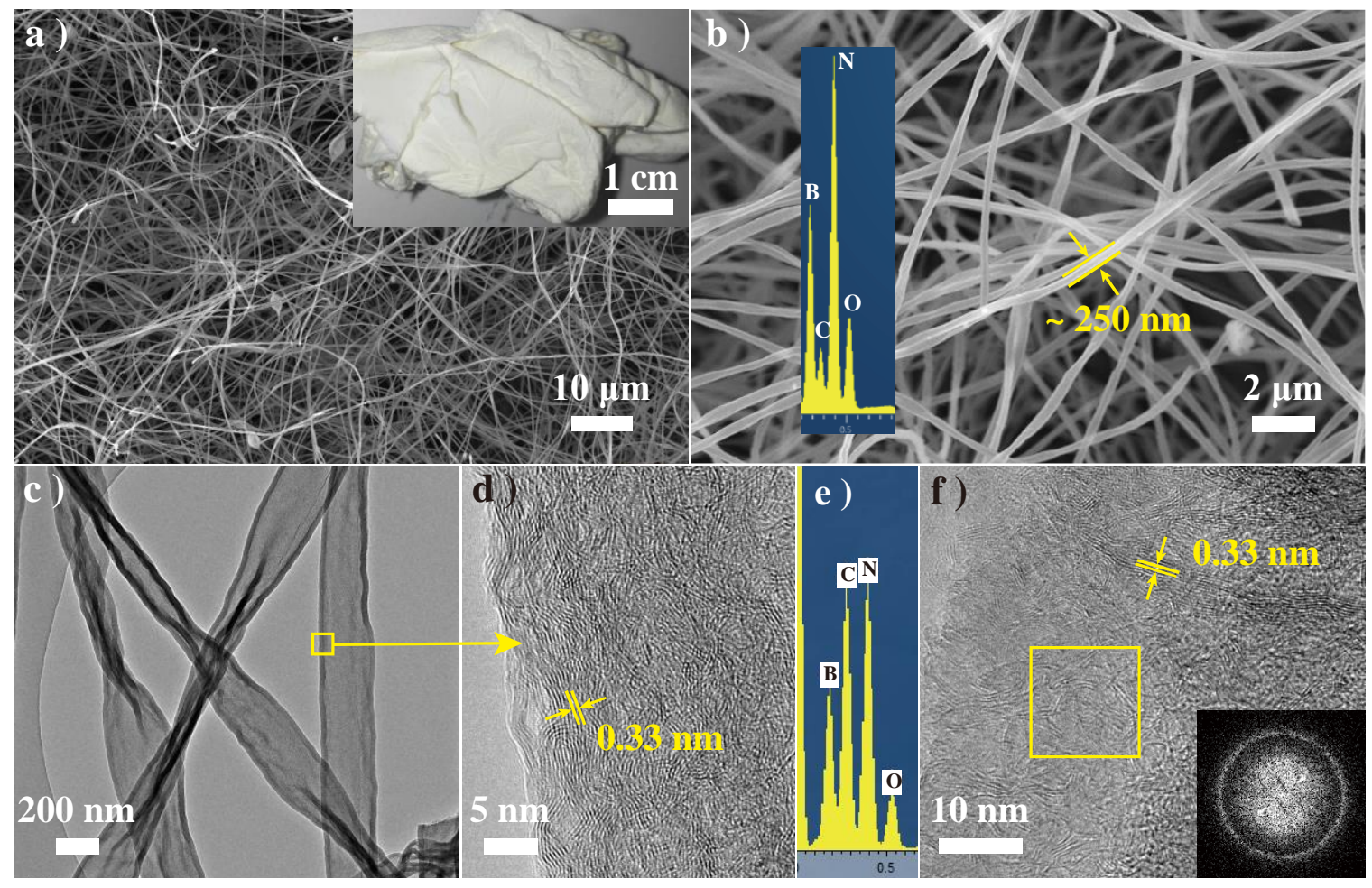

Figure 2. a) Low magnification SEM image and in inset the corresponding digital photograph of the obtained unwoven h-BN nanotube mat; b) SEM image of unwoven BNNTs with a diameter ranging from 150 to $300 \mathrm{~nm}$ and in inset the corresponding EDXS spectrum; c) Low magnification TEM image of the BNNTs; d) High resolution TEM image recorded from the side BNNT wall displaying lattice fringes with typical lattice distance corresponding to c axis of h-BN; e) Corresponding EDXS spectrum with carbon signal arising from the TEM grid; f) High resolution TEM image recorded from the open-end of BNNT with its corresponding area selected (yellow frame) FFT image in inset demonstrated the polycrystalline structure.

Prior to investigate the potential ability of the unwoven BNNTs for water treatment, the wetting properties of unwoven BNNT mat are studied using contact angle (CA) measurements. The fabricated unwoven BNNT mat exhibits excellent water repellence independently of the solution $\mathrm{pH}$ with $\mathrm{CA}$ near $150^{\circ}$, as shown in Figure 3a. Indeed, no significant variation of CA is noted with $\mathrm{pH}$ ranging from 0 to 14 . On the other hand organic solvents such as hexane and dichloromethane are rapidly absorbed by the porous material. On flat BN film on Si wafer, the organic liquid immediately spread over the surface leading to a contact angle of $\sim 0^{\circ}$ (not shown here). The observed super-hydrophobicity is attributed to a synergy effect between intrinsic BN properties and surface roughness of the mat arising from it crosslinked structure. Indeed, both simulations and experiments recently demonstrate the weak hydrophobicity of BN layers due to the polarity of the B-N bond, ${ }^{44,45}$ while modification of the surface roughness of BN thin films lead to the drastic increase of the $\mathrm{CA}$ from $67^{\circ}$ to $159^{\circ} .{ }^{46}$ Vertically grown $\mathrm{BN}$ nanotubes on silicon substrate also display super-hydrophobic bahavior. ${ }^{44,47}$ 
Stability of the material properties over time, storage and use conditions is a key parameter for usability and lifetime of the material. In particular, stability toward air exposure and $\mathrm{pH}$ is examined to evaluate an eventual BN oxidation. After 15 and 30 days of material storage under ambient condition without special care, CA values remain constant, around $150^{\circ}$ over the full $\mathrm{pH}$ range investigated (Figure 3c,d). It can also be noted that after immersion into different $\mathrm{pH}$ solutions for 7 days, no drastic change of deionized water CA is observed (Figure 3b) despite a slight tendency to lower. Excellent stability toward air and $\mathrm{pH}$ in the full range is thus demonstrated. Owing to the fact that BN materials with poor crystallinity will partial oxidized after air exposure, one can safely conclude that high quality BN nanotubes are fabricated using a two-step ALD process.

a )

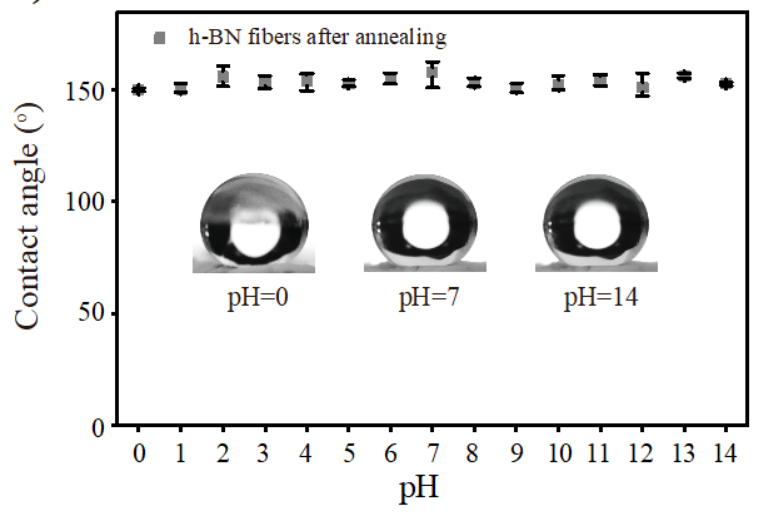

c)

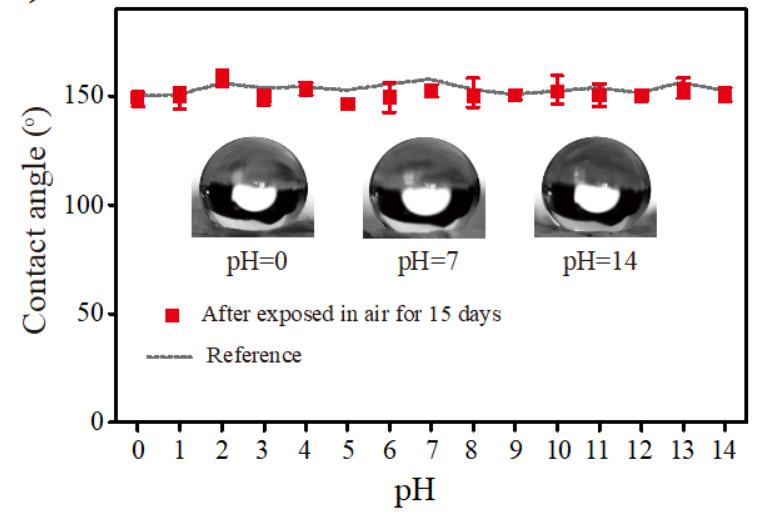

b )

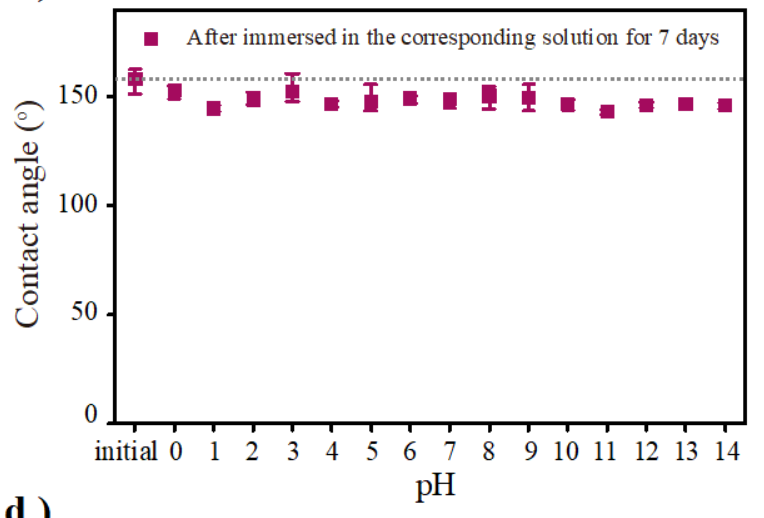

d )

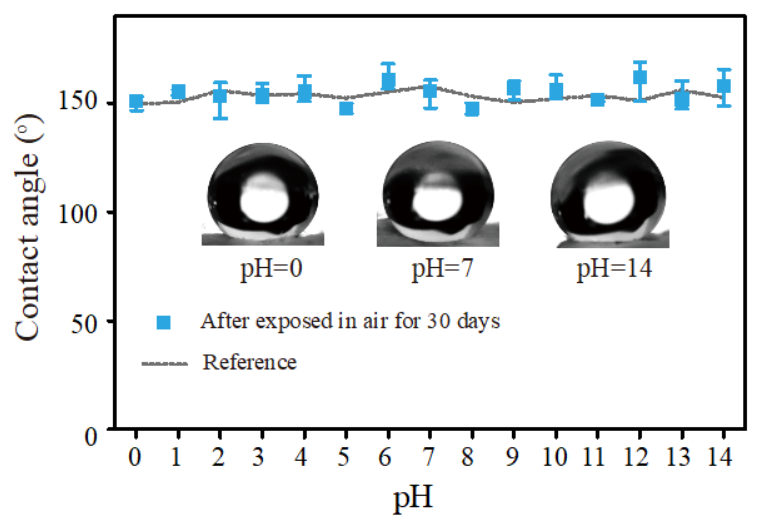

Figure 3. a) Measured contact angles of as-prepared unwoven BNNT mat using aqueous solutions with $\mathrm{pH}$ varying from 0 to 14 ; b) Deionized water contact angles of the BNNTs after their immersion in solutions with various pH value range 0-14 for 7 days; Contact angles as a function of the $\mathrm{pH}$ solution after storage of the mat in air for c) 15 days and d) 30 days. Grey dash lines correspond to the initial data obtained from fresh mat.

\section{Absorption approach for oil/water separation}

In the view of water purification, absorption is an efficient way to remove oils and organic solvents in the polluted water. The ability of BNNT mat to remove various organic compounds from water by their 
absorption is thus evaluated. The evolution of used engine oil soaking process is depicted in Figure 4a-c. The white unwoven mat is first put into a glass dish containing used engine oil floating on top of water (Figure 4a). The oil then quickly soaks into the BNNT material when in contact with it with rapid change of the mat color (Figure 4b). The absorption process finishes within less than $60 \mathrm{~s}$; no additional visual change occurs over time (Figure $\mathbf{4 c}$ ). The mat, floating at the surface, can thus easily be removed. The recovery is then realized using an annealing process partially under $\mathrm{NH}_{3}$ in order to remove the oil while avoiding oxidation of the BN tubes. As visible in Figure 4d,e the unwoven BN mat retains its original macroscopic features after regeneration. No mass loss and shape change are noted, sign of its good stability. In order to verify its reusability, the same mat is employed for 5 successive absorption tests (Figure 4e,f,g), without performance modification noted compare to a fresh mat. Furthermore, asfabricated and regenerated five times BNNTs exhibit both typical FTIR spectra (ESI, Figure S3a) of BN without presence of additional band. TEM imaging confirms maintaining of the original structure as well as of the crystallinity of the BN nanotubes even after 5 regenerations (ESI, Figure S3).

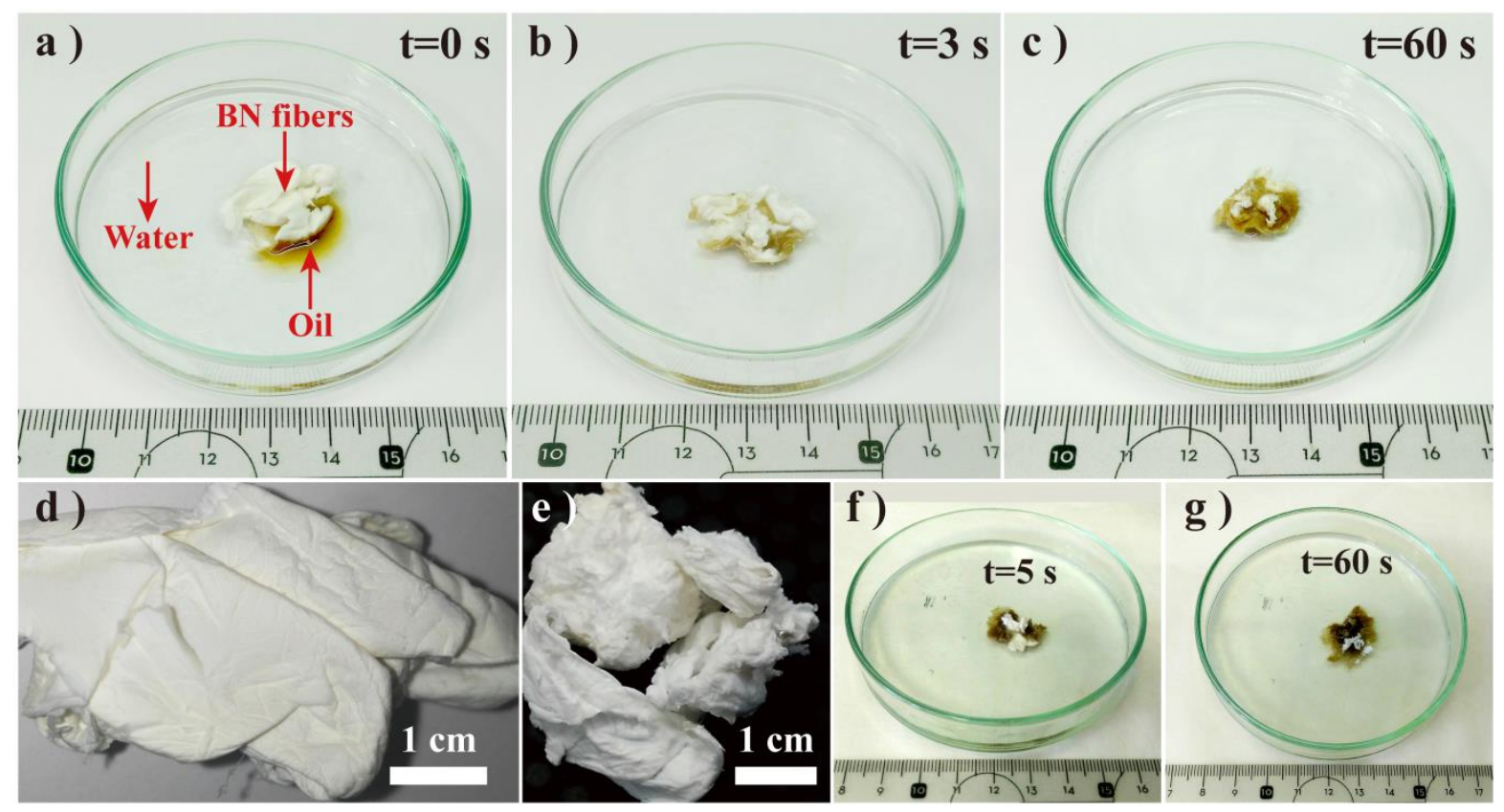

Figure 4. Photographs of used engine oil absorption experiment using a fresh unwoven BNNT mat at a) $t=0 \mathrm{~s}, \mathrm{~b}) \mathrm{t}=3 \mathrm{~s}$ and $\mathrm{c}$ ) $\mathrm{t}$ $=60 \mathrm{~s}$; Photographs of d) as-prepared and e) recovered unwoven BNNT mat; Photographs during the oil absorption experiment using regenerated BNNTs after f) $5 \mathrm{~s}$ and g) $60 \mathrm{~s}$.

The successful full recovery of the mat as well as its reusability without change of its absorption capacities is thus confirmed. Its absorptive capacity toward various oils and organics solvent and the influence of the diameter of the $\mathrm{BN}$ tubes are then investigated, by adding the mats into the corresponding pure compound. 
Two unwoven BNNT mats (Figure 5a,b) with average tube diameter of $\sim 250 \mathrm{~nm}$ and $\sim 500 \mathrm{~nm}$, respectively, are prepared from PAN fibers with the corresponding diameters. The absorptive capacities toward pump, mineral and used engine oil as well as toluene and dichloromethane are evaluated and compared in Figure 5c. Weightings are done rapidly to avoid/minimized evaporation of tested organic solvent, while $5 \mathrm{~s}$ "cleaning" using a tissue are used in the case of oil soaking to ensure that no extra high viscous oil drops could influence the measurements. Much higher loading capacities than that previously reported with $\mathrm{BN}$ nanomaterials ${ }^{17}$ is observed in all cases. Indeed, the unwoven BNNT mats can uptake organic solvents/oils per 50 to 140 times of its initial weight depending in the tube diameter, whereas absorption capacity of maximum 33, 10 and 6 times their own weight is noted for, respectively, porous BN nanosheets, ${ }^{17} \mathrm{BN}$ nanosheet/polyvinylidene fluoride composite and $\mathrm{BN}$ foam like monoliths. Moreover, $500 \mathrm{~nm}$ diameter BNNTs exhibit near twice higher and faster uptake capacity than that with an average diameter of $250 \mathrm{~nm}$. Those results suggest that capacity of the fibers to remove the organic part is governed by the accessible volume and the capillary effect inside the tube. Similar ability or even more efficiency is displayed by these fabricated mats compared to several other materials such as cotton and carbon-based nanostructures, excepted specific structures as ultra-flyweight aerogel. It is worth mentioning also that no direct correlation can be done here between the molecular weight, density, viscosity of the organics and the absorption ability of the mat.

Finally the BN mat can be recovered at least 5 times without significant decrease of its absorption capacities, especially toward dichloromethane and toluene (Figure 5d). Nevertheless, a slight downfall of its oil absorptive ability is noted. One can hypothesize that it might be related to small structural change due to strain and stress generated by the high density of oils. However no evidence of chemical or structural modification has been highlighted for the moment by FTIR and TEM.

The good repeatability/recovery is attributed to the excellent stability of the 3D unwoven BN structure, whereas other activated carbon or polymer based materials display poor repeatability for the absorption. Such material appears high suited for water treatment using soaking principle. 

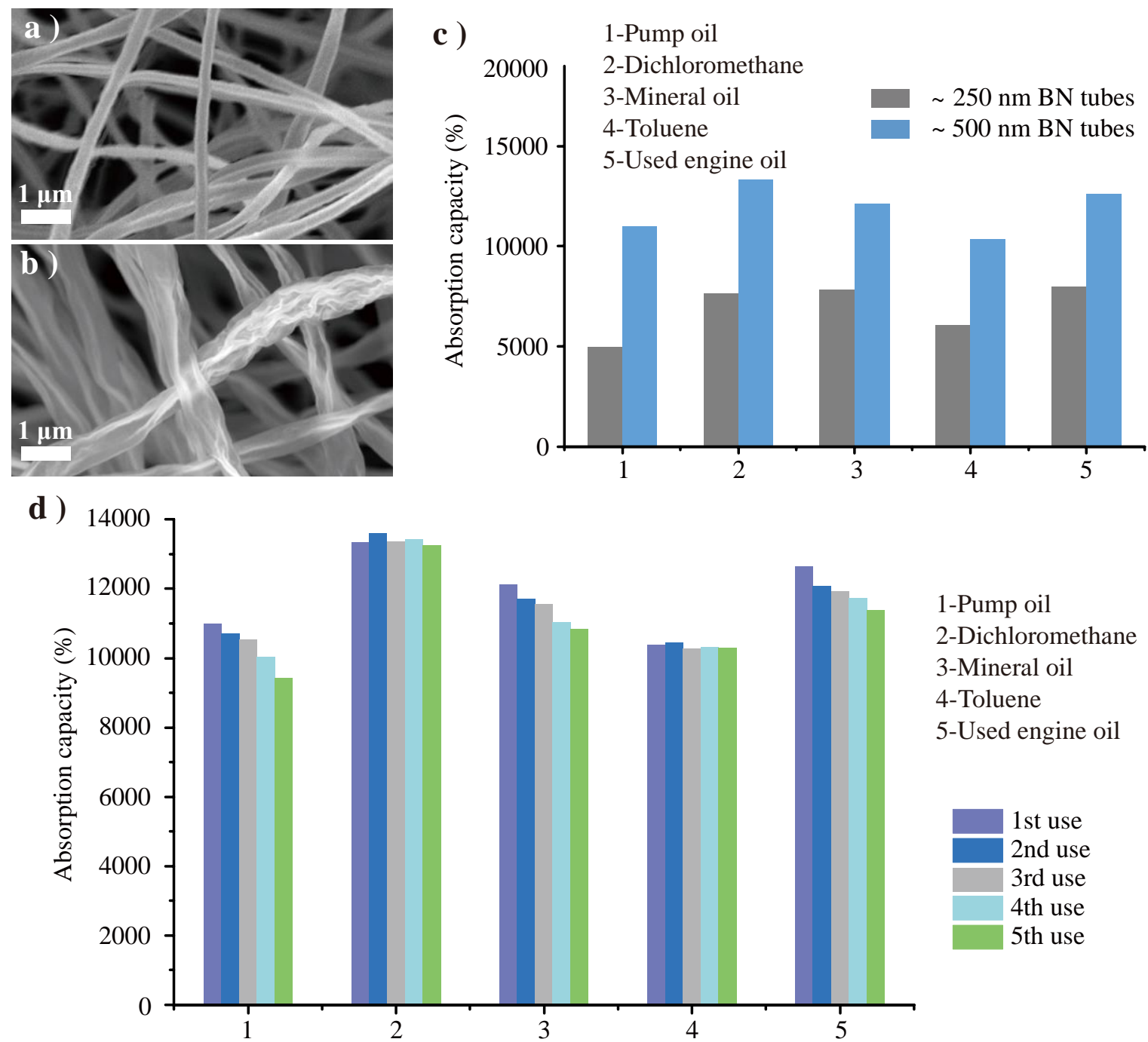

Figure 5. SEM images of the unwoven BNNTs with inner diameter range of a) 150-300 nm and b) 400-600 nm; c) Gravimetric absorption capacities of freshly prepared unwoven mat with two different tube diameters of about $250 \mathrm{~nm}$ and $500 \mathrm{~nm}$ toward five different solvents and oils; d) Reuse ability test of 400-600 nm BNNTs toward the different organics.

\section{Filtration approach for oil/water separation}

Due to its super hydrophobicity and thus high lipophilicity as well as its porous structure, unwoven mat of boron nitride nanotubes appears as a filter of choice. Indeed, BN nanotube coated stainless steel meshes ${ }^{22}$ and $\mathrm{BN}$ nanosheet films displaying designed structures ${ }^{12}$ recently demonstrate promising abilities for immiscible oil/mixture and emulsion separation, respectively. Figure 6 depicts the used home-made filtration system in which the BN material is hold at the tube junction between two large mesh metal grids. Separation of $20 \mathrm{~mL}$ biphasic solution $(\mathrm{V} / \mathrm{V}=15: 5)$ of dichloromethane and soda solution at $\mathrm{pH} 9$ (dyed using bromothymol blue) (Figure 6a,b) is first realized. The lower phase consists in dichloromethane which has a higher density than water. It is thus directly in contact with the filter, facilitating the filtration 
process. Dichloromethane passes quickly through the BNNT mat whereas dyed aqueous solution remains on top of the filter even after $30 \mathrm{~min}$. The collected volume of each separated phase corresponds to $14 \mathrm{~mL}$ of dichloromethane and $4.8 \mathrm{~mL}$ of aqueous solution (Figure 6c,d). The $1 \mathrm{~mL}$ volume loss of organic phase is most probably due to the fast evaporation of the chemical, while $0.2 \mathrm{~mL}$ loss of water is attributed to transfer process and measurement uncertainty. It is noteworthy that the separation process of $20 \mathrm{~mL}$ biphasic solution can reproducibly be repeated at least 10 times. The filter is washed with dichloromethane and dried between each filtration. No residue is observed in the collected organic solvent and water, demonstrating the high separation efficiency of the unwoven BNNTs.
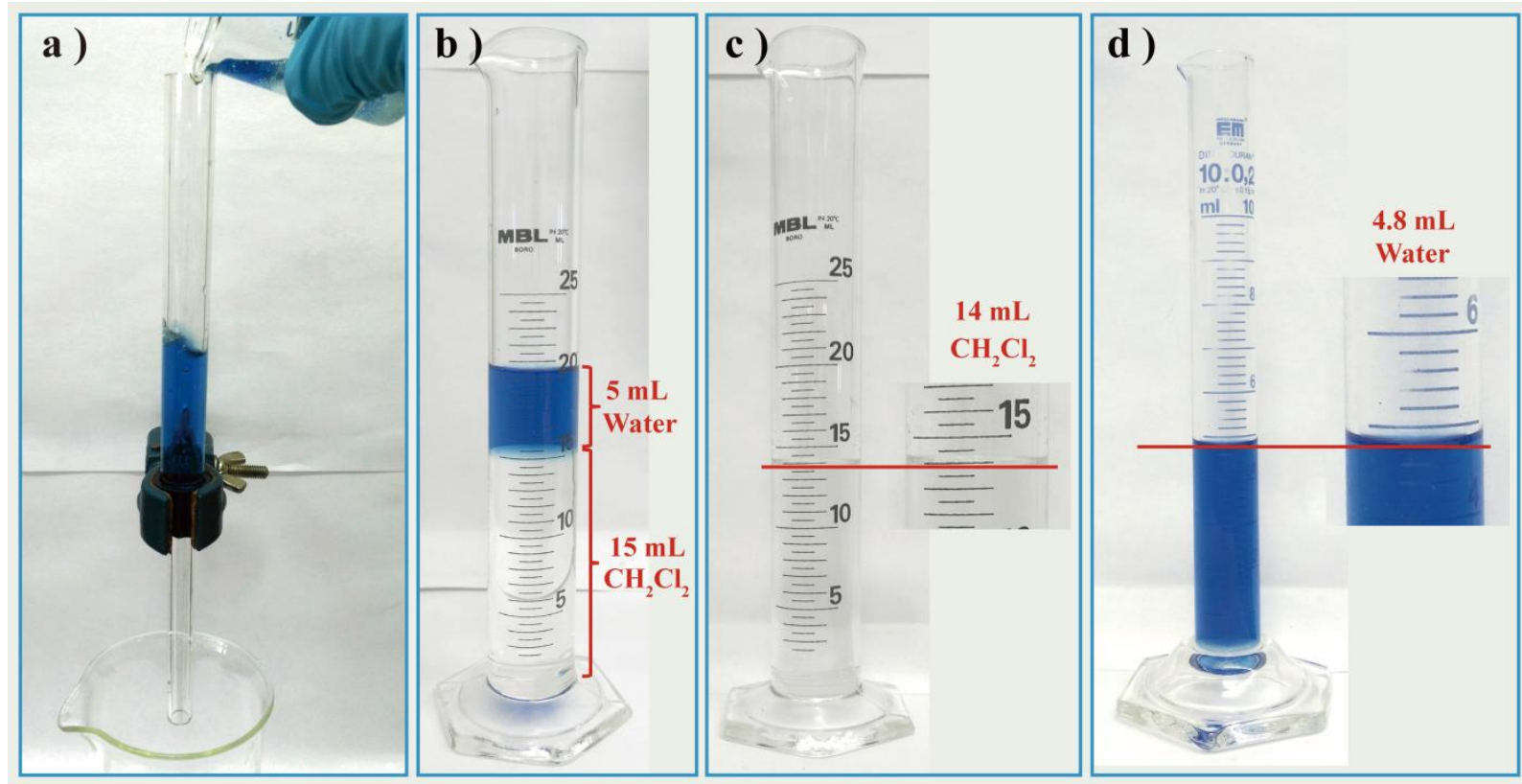

Figure 6. Photographs of a) the experimental filtration system, b) the feed dichloromethane/water solution before filtration, c) the filtrated dichloromethane d) and water collected after filtration.

While separation of two immiscible liquids can appear straightforward, water purification of a stable emulsion is more challenging and of great interest. Therefore, filtration of stable emulsion of water in chloroform ( $\mathrm{V} / \mathrm{V}=1: 100)$ with surfactant is studied. The size distribution of the water droplets in the emulsion is characterized by optical microscopy (Figure 7a,b). Due to fast evaporation (in $3 \mathrm{~s}$ ) of chloroform, the particle size is determined from the remaining water droplets on glass slide (Figure 7b). The particle diameter ranges from 2 to $16 \mu \mathrm{m}$ that is larger than BNNT diameter. Using the same filtration setup than previously, the water contained in the turbid solution is successfully separated from the chloroform solvent. The whitish feed solution (Figure 7c, left) turns indeed transparent (Figure 7c right) once filtrated, as nicely shown by the photograph in the Figure 7c. Optical microscope image of the filtrate (Figure 7d) confirms the absence of water particle after filtration. It should be mentioned that the 
emulsion is stable in absence of filtration as shown in Figure 7e, no demulsification occurring at least within 7 days.

The separation process is repeated for at least 5 times using the same filter without loss of efficiency. As for biphasic solution, unwoven $\mathrm{BN}$ nanotube mat appears an excellent reusable filter for emulsion purification. However, it is worth noting that the separating process of emulsion is rather slow. Using 0.5 $\mathrm{cm}$ x $0.5 \mathrm{~cm}$ filter, separation of $20 \mathrm{~mL}$ solution under atmospheric pressure lasts $10 \mathrm{~min}$. Such emulsion separation is expected to be accelerated using either larger filter and/or vacuum condition.

Unwowen BN mats, despite their random porosity, demonstrate good capability as filter for water purification such as previously used BN structures that display organized/designed texture. ${ }^{12} 22$
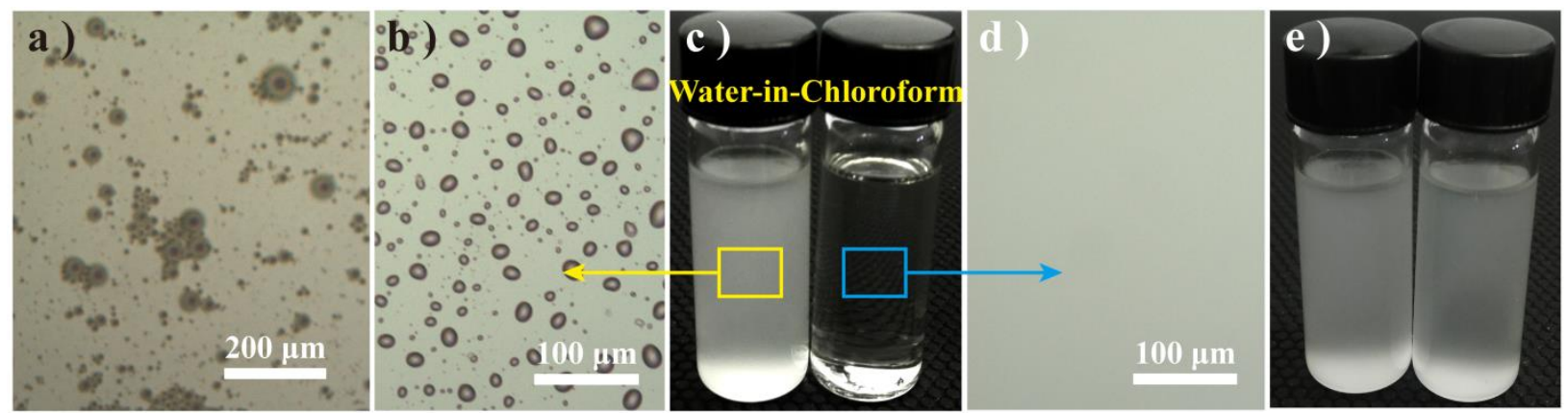

Figure 7. Optical microscope images of emulsion a) as deposited on glass slide and b) after chloroform evaporation c) Photograph of as prepared water-in-chloroform emulsion (left) and obtained filtrate (right); d) Optical microscope image of the filtrate does not display presence of water droplets; e) Photograph of the freshly-prepared emulsion (left) and after prepared 7 days without stirring (right).

Risk of a second contamination by the nanomaterials used for the water purification being an important issue, presence of $\mathrm{BN}$ material is verified by SEM in each purified water phase, remaining on top of the filter. $15 \mu \mathrm{L}$ of water is thus dropped onto clean silicon substrates. In all cases, no BNNT is detected in the SEM images (not shown).

\section{Conclusions}

In summary, unwoven BNNTs are fabricated from low cost polymer template using a recent two-step ALD process. The obtained boron nitride mat presents high crystallinity and oxidation resistance. They exhibit outstanding hydrophobicity and lipophilicity properties as well as exceptional stability over a long period. Neither air exposure nor drastic $\mathrm{pH}$ conditions alter noticeably their properties. Furthermore, BNNTs show high capacity of oil and organic solvent absorption as well as excellent recovery. Importance of the nanotube size toward its soaking properties is demonstrated. The fabricated boron nitride mat reveals to be a highly efficient and renewable filter for separating biphasic oil/water solution 
and water-in-oil emulsion. Very good repeatability of such filtration is observed without noticeable second contamination risk. Therefore these unwoven BNNT mats are highly suitable for a wide range of applications in water purification. They display suited mechanical resistance and thus large scale treatment system can be foreseen. The low temperature first fabrication step allows coating of wide range of materials such as a variety of unwoven and woven clothes that are easy to handle and highly suited for purification systems.

\section{Associated content}

\section{Electronic Supporting Information}

The Supporting Information is available: Photographs of the home-made filtration system used with a) biphasic solution and b) emulsion. SEM image of the original PAN fibers with average diameter of a) 500 and b) $250 \mathrm{~nm}$. a) FTIR spectra recorded from freshly prepared (black) and five times regenerated unwoven (red) BNNT mat unwoven. b-d) TEM images of unwoven BNNTs after the $5^{\text {th }}$ recovery: b) low magnification TEM image; high resolution TEM image c) of a tube wall and d) of one nanotube end with in inset the corresponding FFT image.

\section{Conflict of Interest}

There are no conflicts of interest to declare.

\section{Acknowledgements}

The authors thank the "Centre Technologique des Microstructures", CT $\mu$ (Université Lyon 1), for providing access to the TEM and SEM facilities. V. Salles is kindly acknowledged for the electrospun PAN fibers.

This work has been financially supported by the National Research Agency, France (project $\mathrm{n}^{\circ}$ ANR-16CE08-0021-01). W. Hao acknowledges the China Scholarship Council (CSC) for the PhD grant support.

\section{References}

1 H. Sadegh, G. A. M. Ali, V. K. Gupta, A. S. H. Makhlouf, R. Shahryari-ghoshekandi, M. N. Nadagouda, M. Sillanpaa and E. Megiel, J. Nanostructure Chem., 2017, 7, 1-14.

2 Y. Ying, W. Ying, Q. Li, D. Meng, G. Ren, R. Yan and X. Peng, Appl. Mater. Today, 2017, 7, $144-158$.

3 C. H. Lee, B. Tiwari, D. Zhang and Y. K. Yap, Env. Sci Nano, 2017, 4, 514-525.

4 Z. Xu, Y. Zhao, H. Wang, H. Zhou, C. Qin, X. Wang and T. Lin, ACS Appl. Mater. Interfaces, 2016, 8, 56615667. 
5 P. H. Krishnamurthy, L. T. Yogarathinam, A. Gangasalam and A. F. Ismail, J. Appl. Polym. Sci., 2016, 133, 43873.

6 S. Bolisetty and R. Mezzenga, Nat. Nanotechnol., 2016, 11, 365-371.

7 W. Zhang, N. Liu, Y. Cao, Y. Chen, L. Xu, X. Lin and L. Feng, Adv. Mater., 2015, 27, 7349-7355.

8 R. Das, M. E. Ali, S. B. A. Hamid, S. Ramakrishna and Z. Z. Chowdhury, Desalination, 2014, 336, 97-109.

9 H. Y. Yang, Z. J. Han, S. F. Yu, K. L. Pey, K. Ostrikov and R. Karnik, Nat. Commun., 2013, 4, 2220.

10 P. Singla, N. Goel, V. Kumar and S. Singhal, Ceram. Int., 2015, 41, 10565-10577.

11 G. Lian, X. Zhang, H. Si, J. Wang, D. Cui and Q. Wang, ACS Appl. Mater. Interfaces, 2013, 5, 12773-12778.

12 T. Li, L. Wang, K. Zhang, Y. Xu, X. Long, S. Gao, R. Li and Y. Yao, Small, 2016, 12, 4960-4965.

13 Q. Weng, X. Wang, X. Wang, Y. Bando and D. Golberg, Chem Soc Rev, 2016, 45, 3989-4012.

14 A. Pakdel, C. Zhi, Y. Bando, T. Nakayama and D. Golberg, ACS Nano, 2011, 5, 6507-6515.

15 J. Yu, L. Qin, Y. Hao, S. Kuang, X. Bai, Y.-M. Chong, W. Zhang and E. Wang, ACS Nano, 2010, 4, 414-422.

16 Y. Xue, P. Dai, X. Jiang, X. Wang, C. Zhang, D. Tang, Q. Weng, X. Wang, A. Pakdel, C. Tang, Y. Bando and D. Golberg, J Mater Chem A, 2016, 4, 1469-1478.

17 W. Lei, D. Portehault, D. Liu, S. Qin and Y. Chen, Nat. Commun., 2013, 4, 1777.

18 J. Li, X. Xiao, X. Xu, J. Lin, Y. Huang, Y. Xue, P. Jin, J. Zou and C. Tang, Sci. Rep., 2013, 3, 3208.

19 J. Li, J. Lin, X. Xu, X. Zhang, Y. Xue, J. Mi, Z. Mo, Y. Fan, L. Hu, X. Yang, J. Zhang, F. Meng, S. Yuan and C. Tang, Nanotechnology, 2013, 24, 155603.

20 F. Liu, J. Yu, X. Ji and M. Qian, ACS Appl. Mater. Interfaces, 2015, 7, 1824-1832.

21 J. Li, H. Jia, J. Lin, H. Luo, Z. Liu, X. Xu, Y. Huang, P. Jin, J. Zhang, S. Abbas and C. Tang, RSC Adv, 2015, 5, $71537-71543$.

22 Y. Yu, H. Chen, Y. Liu, V. Craig, L. H. Li and Y. Chen, Adv. Mater. Interfaces, 2014, 1, 1300002.

23 M. A. Rafiee, T. N. Narayanan, D. P. Hashim, N. Sakhavand, R. Shahsavari, R. Vajtai and P. M. Ajayan, Adv. Funct. Mater., 2013, 23, 5624-5630.

24 D. Liu, L. He, W. Lei, K. D. Klika, L. Kong and Y. Chen, Adv. Mater. Interfaces, 2015, 2, 1500228.

25 Y. Yu, H. Chen, Y. Liu, V. S. J. Craig, C. Wang, L. H. Li and Y. Chen, Adv. Mater. Interfaces, 2015, 2, 1400267.

26 L. H. Li, Y. Chen and A. M. Glushenkov, J. Mater. Chem., 2010, 20, 9679-9683.

27 L. H. Li and Y. Chen, Langmuir, 2010, 26, 5135-5140.

28 L. B. Boinovich, A. M. Emelyanenko, A. S. Pashinin, C. H. Lee, J. Drelich and Y. K. Yap, Langmuir, 2012, 28, 1206-1216.

29 M. Knez, K. Nielsch and L. Niinistö, Adv. Mater., 2007, 19, 3425-3438.

30 C. Marichy, M. Bechelany and N. Pinna, Adv. Mater., 2012, 24, 1017-1032.

31 M. Leskelä and M. Ritala, Angew. Chem. Int. Ed., 2003, 42, 5548-5554.

32 W. Hao, C. Marichy, C. Journet and A. Brioude, ChemNanoMat, 2017, 3, 656-663.

33 M. Weber, B. Koonkaew, S. Balme, I. Utke, F. Picaud, I. Iatsunskyi, E. Coy, P. Miele and M. Bechelany, ACS Appl. Mater. Interfaces, 2017, 9, 16669-16678.

34 Y. Yu, H. Chen, Y. Liu, V. S. J. Craig and Z. Lai, Adv. Colloid Interface Sci., 2016, 235, 46-55.

35 G. Constant and R. Feurer, J. Common Met., 1981, 82, 113-118. 
36 V. Salles, S. Bernard, A. Brioude, D. Cornu and P. Miele, Nanoscale, 2010, 2, 215-217.

37 S. Cavaliere, V. Salles, A. Brioude, Y. Lalatonne, L. Motte, P. Monod, D. Cornu and P. Miele, J. Nanoparticle Res., 2010, 12, 2735-2740.

38 B. Tiwari, D. Zhang, D. Winslow, C. H. Lee, B. Hao and Y. K. Yap, ACS Appl. Mater. Interfaces, 2015, 7, 26108-26116.

39 G. N. Parsons, S. E. Atanasov, E. C. Dandley, C. K. Devine, B. Gong, J. S. Jur, K. Lee, C. J. Oldham, Q. Peng, J. C. Spagnola and P. S. Williams, Coord. Chem. Rev., 2013, 257, 3323-3331.

40 S.-M. Lee, E. Pippel, U. Gösele, C. Dresbach, Y. Qin, C. V. Chandran, T. Bräuniger, G. Hause and M. Knez, Science, 2009, 324, 488-492.

41 K. E. Gregorczyk, D. F. Pickup, M. G. Sanz, I. A. Irakulis, C. Rogero and M. Knez, Chem. Mater., 2014, 27, 181-188.

42 S.-M. Lee, V. Ischenko, E. Pippel, A. Masic, O. Moutanabbir, P. Fratzl and M. Knez, Adv. Funct. Mater., 2011, 21, 3047-3055.

43 M. Bechelany, S. Bernard, A. Brioude, D. Cornu, P. Stadelmann, C. Charcosset, K. Fiaty and P. Miele, J. Phys. Chem. C, 2007, 111, 13378-13384.

44 C. H. Lee, J. Drelich and Y. K. Yap, Langmuir, 2009, 25, 4853-4860.

45 H. Li and X. C. Zeng, ACS Nano, 2012, 6, 2401-2409.

46 G.-X. Li, Y. Liu, B. Wang, X.-M. Song, E. Li and H. Yan, Appl. Surf. Sci., 2008, 254, 5299-5303.

47 L. B. Boinovich, A. M. Emelyanenko, A. S. Pashinin, C. H. Lee, J. Drelich and Y. K. Yap, Langmuir, 2011, 28, 1206-1216.

48 J. Aurell and B. K. Gullett, Environ. Sci. Technol., 2010, 44, 9431-9437.

49 X. Gui, J. Wei, K. Wang, A. Cao, H. Zhu, Y. Jia, Q. Shu and D. Wu, Adv. Mater., 2010, 22, 617-621. 